\title{
Etude et modélisation sous Simulink d'une chaîne de transmission DVB-S
}

\author{
Lilian BOSSUET, Guillaume FERRE \\ ENSEIRB - Département Electronique \\ 1, avenue du Dr Albert Schweitzer - BP 99 - 33402 TALENCE Cedex - France \\ Email : lilian.bossuet@enseirb.fr \& guillaume.ferre@enseirb.fr
}

\begin{abstract}
Résumé
La modélisation informatique de systèmes de communications est un moyen efficace et rapide pour mettre en lumière les performances et les principales difficultés de conception de ces derniers. En se servant de bibliothèques, un outil de simulation comme Simulink permet de modéliser de façon très pédagogique des chaînes de transmissions, pour en analyser par exemple les performances en termes de taux d'erreur binaire (TEB). C'est ainsi que nous proposons aux étudiants de tout d'abord analyser et comprendre la norme DVB-S, pour en modéliser la transmission au niveau de la couche physique. La réalisation de cet enseignement passe donc par trois étapes principales. En effet, dans un premier temps, nous fournissons aux étudiants seulement la norme DVB-S. Cela leur permet alors de voir comment est rédigée une norme et d'en extraire le maximum d'informations pour modéliser la couche physique. Au bout d'une heure, nous leur distribuons une synthèse contenant les principaux paramètres de la norme (codeur interne, codeur externe, entrelaceur/désentrelaceur, taille des paquets, débits, bande passante...), puis au travers de quelques questions, nous leur faisons réaliser pas à pas la chaîne de transmission complète.
\end{abstract}

\section{Mots clés}

Communications numériques par satellite, modélisation Simulink, étude d’une norme.

\section{Introduction}

L'étude, la conception, le développement et la production de produits technologiques s'appuient très largement sur l'utilisation de normes internationales (normes OSI). L'ingénieur doit donc être capable d'utiliser ces normes. Celles-ci se trouvent dans tous les domaines technologiques, aussi il est indispensable durant la formation des jeunes étudiants ingénieurs de les mettre en face de documents de normes et les amener à appréhender ces outils de travail. C'est ce que nous nous proposons de faire à travers ce projet qui concerne l'étude et la simulation d'une chaîne de transmission DVB-S (Digital Video Broadcasting by Satellite) à partir de la norme européenne éditée par l’ETSI [1-2].

L'évolution croissante des télécommunications par satellite a conduit à une normalisation des systèmes de communications, la norme DVB-S fait partie de ces normes pour la transmission de vidéo. Cette norme est basée sur l'échange de données au format MPEG-2 en utilisant une liaison par satellite. D'autres normes, comme la norme DVB-C pour le câble et la norme DVB-T pour les émetteurs terrestres, traitent de l'échange de fichier du même type mais dans des canaux de communications différents.

La compréhension et la mise en œuvre de la norme DVB-S pour un étudiant ingénieur en filière électronique numérique n'est pas simple. Aussi pour permettre aux étudiants de mener à bien ce projet de modélisation de la norme avec le logiciel Simulink, nous proposons une approche didactique. Les étudiants, en binôme, progressent par palier dans la modélisation et vérifient à chaque étape la correspondance avec la norme par le biais de métriques caractéristiques comme l'évaluation du taux d'erreur binaire (ou TEB) par exemple. A travers ce projet nous souhaitons développer les 
compétences d'autonomie, de modélisation et d'abstraction des étudiants, afin de les préparer au mieux aux attentes de leurs futures fonctions.

Après avoir présenté la formation à l'ENSEIRB et plus particulièrement la spécialité Système de Radio et Télécommunications (SRT) nous ferrons une présentation pédagogique de ce projet. Celle-ci nous permettra de montrer la finalité de celui-ci vis à vis de la formation des élèves ingénieurs en dernière année de formation. Puis une présentation technique du projet permettra de donner les éléments indispensables à la compréhension du projet et à sa mise en œuvre. Enfin nous exposerons le déroulement du projet et nous donnerons les principaux résultats de simulations.

\section{L'option SRT (Systèmes de Radio et Télécommunications) du département électronique de l'ENSEIRB}

Le projet pédagogique de l'ENSEIRB (Ecole Nationale Supérieure d'Electronique, Informatique et Radiocommunications de Bordeaux) [3] est de former des ingénieurs aux compétences multiples et opérationnelles, aptes à s'épanouir dans leur vie professionnelle et à devenir des éléments moteurs du développement de leur entreprise. L'ENSEIRB s'efforce ainsi de concilier, dans ses objectifs de formation, le développement des deux qualités fondamentales de l'ingénieur d'aujourd'hui : l'efficacité immédiate pour les entreprises et l'indispensable adaptabilité aux mutations technologiques.

Parmi les quatre départements de l'école, le département électronique offre une formation couvrant les principaux domaines de l'électronique, de l'analogique au numérique, auxquels on peut ajouter l'apprentissage des approches systèmes, indispensables pour les nouvelles technologies. Les principaux secteurs visés par cette formation sont la micro-électronique, les équipements de télécommunications, les équipements informatiques et multimédia, l'aéronautique, l'automobile et l'instrumentation. Les élèves de ce département choisissent en dernière année une spécialité. Parmi les différentes possibilités, la spécialité Système de Radio et Télécommunication (SRT) apporte aux étudiants une connaissance technique approfondie des technologies, des circuits et des systèmes pour les télécommunications. Nous développons aussi leurs compétences sur la conception et la caractérisation de ces systèmes, et nous les formons aux méthodes d'analyse de la qualité et de la sécurité des transmissions. Ainsi les élèves disposent en fin de formation de deux double compétences, analogique et numérique, radiocommunications et télécommunications.

Durant le semestre de spécialisation SRT, les étudiants reçoivent de nombreux enseignements théoriques et pratiques du domaine concerné, comme : les techniques avancées de modulations, les standards et protocoles de communications, la structure des objets communicants, les architectures d'émissions/réceptions et les principales fonctions intégrables, le traitement numérique des données, la sûreté de fonctionnement et la fiabilité, ainsi que de nombreux enseignements plus larges de culture de l’ingénieur et de langues vivantes.

\section{Présentation pédagogique du projet}

Le but pédagogique de ce projet est de développer l'autonomie des étudiants et de leur faire concrètement étudier une norme de télécommunications. Nous avons choisi la norme DVB-S [4] plus simple à mettre en œuvre sous Simulink que son évolution DVB-S2 ou encore que la DVB-T [5].

Dans le cadre de ce projet, nous nous adressons à des étudiants en fin de formation, c'est pourquoi l'encadrement durant le projet est le plus léger possible. Dans un premier temps les étudiants suivent souvent une approche empirique, qui par essais successifs leur donne tout d'abord de bons résultats mais qui les conduits rapidement à une impossibilité de pousser plus loin la modélisation dès que le modèle devient complexe. C'est en se rapprochant de la norme et en étudiant bloc après bloc, comme nous le ferons dans la section 4, que les étudiants réussissent à modéliser la chaîne complète d'émission et de réception.

Les étudiants en binôme doivent modéliser le système complet, pour cela ils disposent de 16 heures de travaux pratiques encadrées. Les salles de projet sont en libre accès pour les étudiants qui souhaitent travailler en dehors des heures encadrées. Bien que regroupé en binôme, nous encourageons les 
étudiants à échanger des informations et réfléchir en équipe aux solutions à apporter aux problèmes rencontrés durant le projet.

Les étudiants concernés par ce projet sont en fin de formation d'ingénieur, ils ont une bonne maitrise du logiciel Matlab-Simulink. En deuxième année par exemple, durant 12H00 de TP, ils modélisent les modulations numériques (BPSK, QPSK etc.) directement en code Matlab. C'est pourquoi dans ce projet nous nous appuyons sur ces connaissances pour passer à un niveau d'abstraction plus élevé et nous utilisons les blocs préconçus de la librairie Telecommunication Toolbox de Matlab-Simulink.

La notation du projet prend en compte plusieurs points, l'attitude de l'étudiant durant le projet, son autonomie et un rapport écrit par binôme présentant les principaux résultats.

\section{Présentation technique du projet : la norme DVB-S}

\subsection{Introduction au DVB}

DVB signifie Digital Video Broadcasting. C'est un standard de communication basé sur le MPEG-2 (Moving Picture Experts Group). DVB définit comment transmettre des données au format MPEG-2 en utilisant le satellite (DVB-S [4]), le câble (DVB-C [6]) et la diffusion terrestre (DVB-T [7]).

A l'exception des Etats-Unis d'Amérique, du Mexique, du Canada et de la Corée du Sud, le DVB a été adopté par tous les pays dans le monde pour la télévision et la radio numérique. Les travaux que nous présentons ne traitent que du DVB-S.

La norme DVB-S est l'application de la norme DVB aux transmissions par satellite. Cette norme tient compte des caractéristiques d'une transmission satellitaire, à savoir :

- La bande disponible est relativement large : de 26 à $36 \mathrm{MHz}$,

- Le canal est de type AWGN (Additive White Gaussian Noise),

- Le signal est fortement atténué et dominé par le bruit,

- La transmission est en ligne directe.

Il faut donc mettre en œuvre une transmission efficace à faible rapport signal à bruit. On utilise pour cela, une modulation QPSK associée à un schéma de codage évolué : un codeur de Reed-Solomon (RS) suivi d'un entrelaceur et d'un codeur convolutif (efficace pour réduire le taux d'erreur). En effet le schéma de codage de canal du DVB-S est très particulier et il porte le nom de schéma de codage concaténé. Ainsi du côté récepteur, le premier décodeur (inner decoder) corrige les erreurs en sortie du démodulateur (code convolutif). Le décodeur de sortie (outer decoder), de type RS, a pour but de corriger les rafales occasionnelles d'erreurs introduites par le premier décodeur, qui lui est classiquement un décodeur de Viterbi. En effet, le décodeur de Viterbi produit des rafales d'erreurs (bursts) dans le flot de bits (bitstream) dont la longueur dépasse généralement la capacité de correction du décodeur RS. Afin d'éviter ce problème et d'améliorer les performances de ce codage concaténé on introduit un entrelaceur (interleaver) entre la sortie du codeur RS et l'entrée du codeur convolutif. Ainsi, au niveau du récepteur, la sortie du décodeur convolutif est suivie d'un désentrelaceur (deinterleaver) permettant de répartir l'erreur sur plusieurs mots du code RS. La figure 1 montre les éléments de la chaîne d'émission et de réception. Comme nous le voyons sur cette figure de nombreux blocs élémentaires rentrent en jeux dans cette chaîne, c'est pourquoi le paramétrage complet de la chaîne est compliqué et demande une démarche rigoureuse.

Les canaux de communications satellitaires étant plutôt bruités, le signal transmis est à ce titre, sujet à un grand nombre d'erreurs. Comme les transmissions satellites sont de type broadcast, le récepteur ne peut pas envoyer un message à l'émetteur pour dire « Je n'ai pas reçu le dernier message, pouvez-vous le retransmettre ! ».

On utilise pour cela le FEC (Forward Error Correction). C'est-à-dire que l'émetteur envoie, avec le signal, des informations pour la correction d'erreurs, dans le but de permettre au récepteur de reconstituer le flot de bits (si des erreurs se produisent). Le FEC utilisé avec la modulation QPSK comporte deux formes de correction d'erreurs. La première correction utilise l'algorithme de Viterbi, 
qu'on associe à une fraction (par exemple 2/3). Cette fraction définit la quantité de débit symbole utilisée pour les données réelles, le reste étant utilisé pour la correction d'erreurs. Dans le cas choisi, $2 / 3$ des symboles sont utilisés pour transmettre les données et $1 / 3$ pour transmettre les informations pour la correction d'erreur.

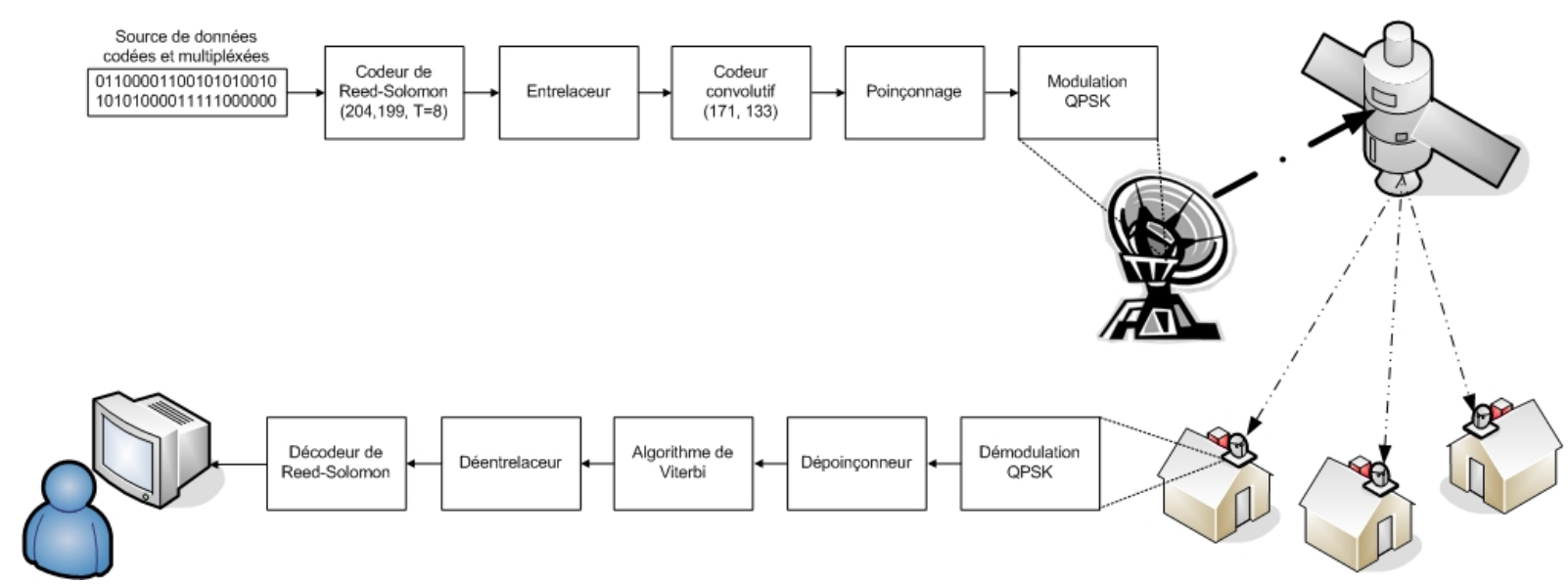

Figure 1 - Chaînes simplifiées d'émission et de réception satellite.

Après avoir réalisé l'algorithme de Viterbi, la seconde forme de correction d'erreurs est relative au codage de Reed-Solomon. En effet, sur 204 octets transmis, 188 contiennent des données et les 16 octets restant sont utilisés comme bits de parités pour aider à corriger les éventuelles erreurs restantes.

Le principe du FEC utilise aussi l'entrelacement des flux de données, pour limiter l'impact en termes d'erreur d'un bruit intempestif dans le flux de données, de la même façon que les CDs l'utilise pour éviter que des rayures ne produisent des pertes d'informations sonores.

\subsection{Illustration de l'intérêt d'un entrelaceur}

Considérons le message suivant :

\section{Le message est reçu}

Après entrelacement, le message pourrait ressembler à :

\section{çeat sum seLe geres}

Si une erreur devait se produire et effaçait la partie «sum» du message (surlignée en jaune), le message désentrelacé serait :

\section{Le *essage e*t reç*}

On s'aperçoit alors que seuls des caractères isolés (représentés par les astérisques et surlignés en jaune) manquent au message, si ce dernier n’avait pas été entrelacé, il ne serait pas possible de corriger les erreurs du fait du pouvoir de correction limité du décodeur RS.

\subsection{Principaux paramètres d'une chaîne d'émission/réception DVB-S}

Le tableau 1 résume les principales caractéristiques d'une chaîne d'émission-réception DVB-S comme celle présentée schématiquement sur la figure 1.

Les différents blocs composant la chaîne d'émission-réception doivent être configurés de sortes à obtenir une continuité des signaux durant la transmission et d'apporter à cette dernière une robustesse vis-à-vis des erreurs pouvant intervenir durant la communication dans le canal bruité. 
Tableau 1 - Principaux paramètres d'une chaîne DVB-S.

\begin{tabular}{|c|c|}
\hline \multicolumn{2}{|c|}{ Paramètres d'une chaine DVB-S } \\
\hline Codage vidéo & MPEG-2 \\
\hline Codage Audio & MPEG-1 \\
\hline Longueur des paquets transportés & 188 octets \\
\hline Polynôme de brouillage & $1+\mathrm{X}^{14}+\mathrm{X}^{15}$ \\
\hline Codeur de Reed-Solomon & $204,188, \mathrm{~T}=8$ \\
\hline Entrelacement convolutif & 12 blocs \\
\hline Codeur convolutif & 171,133 \\
\hline Modulation & QPSK \\
\hline Roll-off & 0,35 \\
\hline Largeur du canal & de 26 à 36 MHz \\
\hline Débit & 23,7 à $41,5 \mathrm{Mb} / \mathrm{s}$ \\
\hline
\end{tabular}

\section{Déroulement du projet et approche didactique}

Pour mener à bien ce projet de modélisation, nous proposons une démarche par étapes aux étudiants. En partant d'une transmission en visibilité directe, bruitée par un bruit additif blanc suivant un loi de distribution gaussienne (BABG : Bruit Additif Blanc Gaussien), les étudiants modélisent la chaîne d'émission et de réception bloc après bloc, de la modulation QPSK au codeur Reed-Solomon. Systématiquement, les étudiants simuleront la transmission de données et évalueront le TEB qui représente le rapport entre le nombre de bits reçus faux et le nombre de bits transmis. C'est un critère de référence pour comparer les performances d’un système de communications.

Pour permettre aux étudiants de ne pas passer trop de temps à la recherche d'informations dans le document de normalisation, un sujet complémentaire leur est distribué dans lequel figure les informations de la figure 1 et du tableau 1, ainsi que différentes questions (présentée dans les paragraphes 5.1 à 5.7) leurs permettant de modéliser pas à pas la chaîne de transmission.

\subsection{Calcul théorique du TEB}

Le TEB s'exprime en fonction du rapport $\frac{E_{b}}{N_{0}}$, où $E_{b}$ est l'énergie d'un bit et $\mathrm{N}_{0}$ est la densité spectrale de puissance du bruit du canal AWGN. En théorie, pour un signal NRZ bipolaire, on a

$$
\mathrm{TEB}=\frac{1}{2} \operatorname{erfc}\left(\sqrt{\frac{E_{b}}{N_{0}}}\right)
$$

Avec erfc la fonction d'erreur donnée par l'équation ci-dessous, où $E_{s}$ désigne l'énergie d'un symbole et SNR (Signal to Noise Ratio) est le rapport entre l'énergie d'un symbole et la densité spectrale de bruit. La figure 2 représente la forme de cette fonction.

$$
\operatorname{erfc}(x)=1-\operatorname{erf}(x)=1-\frac{2}{\sqrt{\pi}} \int_{0}^{x} \exp \left(-t^{2}\right) d t
$$




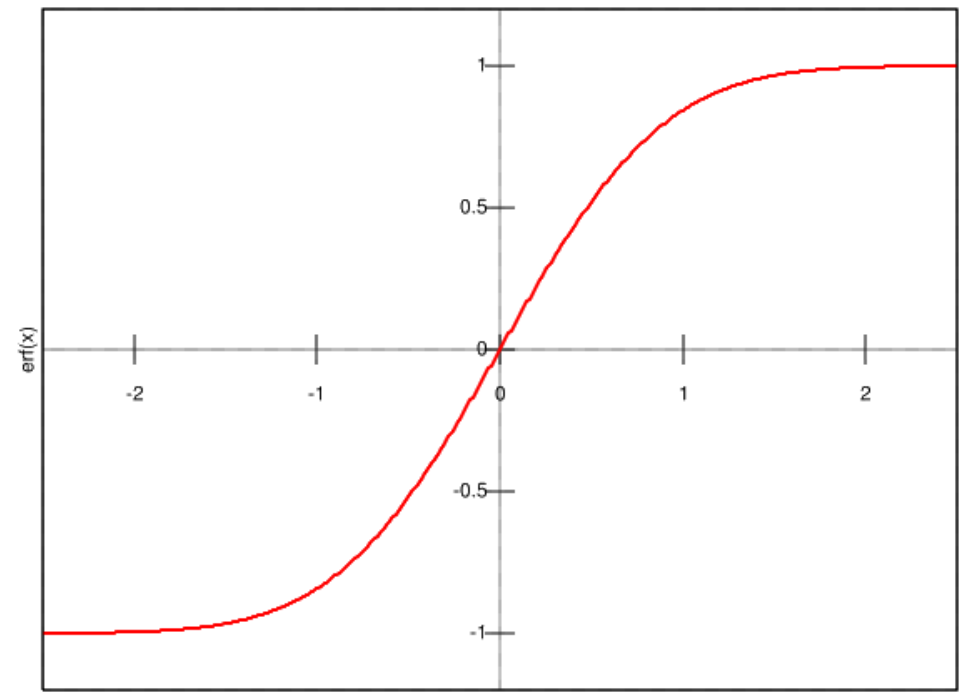

Figure 2 - Forme de la fonction d'erreur ( $x$ en abscisse et erfc $(x)$ en ordonnée).

Dans le cas d'une modulation de type BPSK, un symbole contient un bit. On a donc :

$\mathrm{SNR}=\frac{E_{s}}{N_{0}}=\frac{E_{b}}{N_{0}}$, avec $E_{s}$ l'émergie d'un symbole.

Dans le cas de la modulation QPSK, par contre, un symbole contient deux bits. On a donc :

$\operatorname{SNR}=\frac{E_{s}}{N_{0}}=\frac{2 E_{b}}{N_{0}}$.

En faisant varier le rapport signal sur bruit dans le canal, dans les cas BPSK et QPSK, on obtient les courbes de performances en terme de TEB ci-dessous (figure 3). On constate notament le décalage de $3 \mathrm{~dB}$ entre les performances des deux modulations.

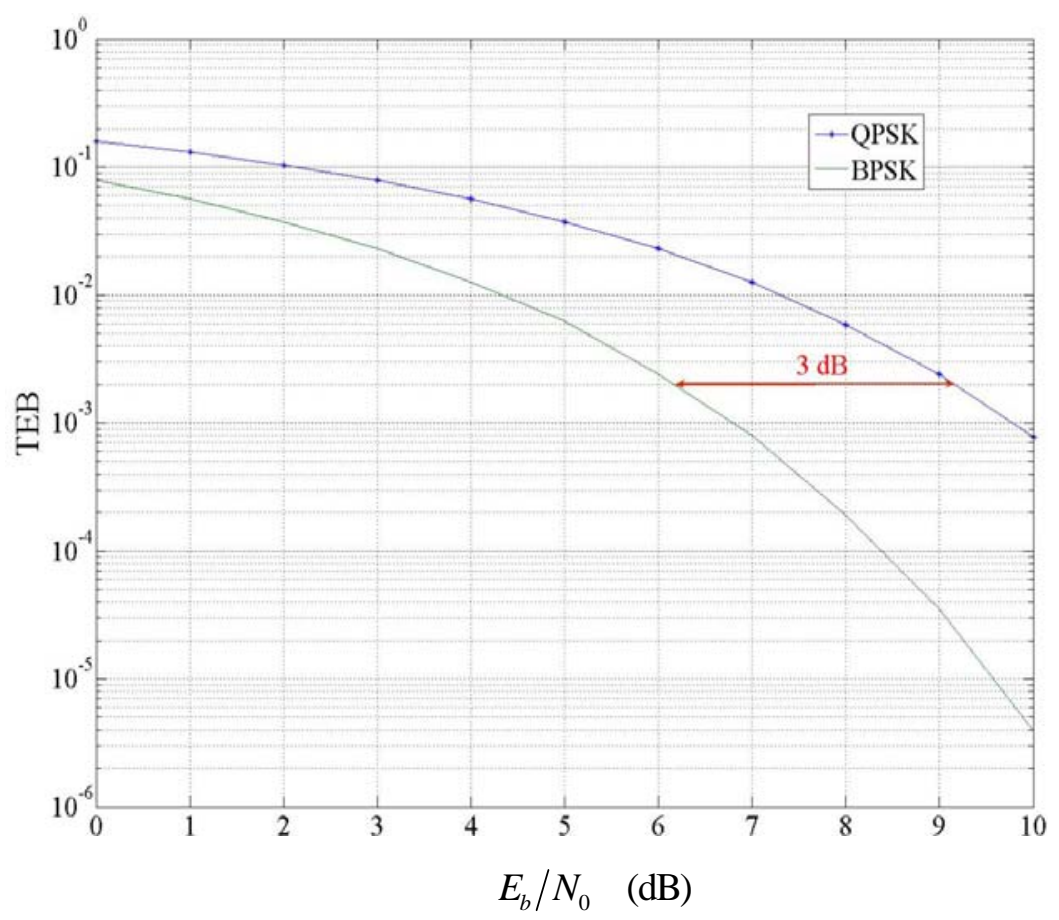

Figure 3 - Evolution du TEB en fonction de $\frac{E_{b}}{N_{0}}$ pour les modulations BPSK et QPSK codées binaires 
Les points de la constellation QPSK ayant une distance plus faible, on constate alors que pour une même énergie symbole $E_{s}$, le TEB pour la modulation QPSK augmente de $3 \mathrm{~dB}$ (facteur 2 que l'on retrouve dans l'expression du SNR précédent).

\subsection{Modélisation de la chaîne de modulation-démodulation}

Dans un premier temps nous proposons aux étudiants de modéliser autour d'un canal AWGN, les blocs de modulation et démodulation, afin de s'initier au logiciel Simulink et à l'utilisation de la Communication Toolbox [8] du logiciel Matlab. La figure 4 montre par exemple la modélisation de la chaîne de modulation avec une modulation de type BPSK et la vérification du TEB.

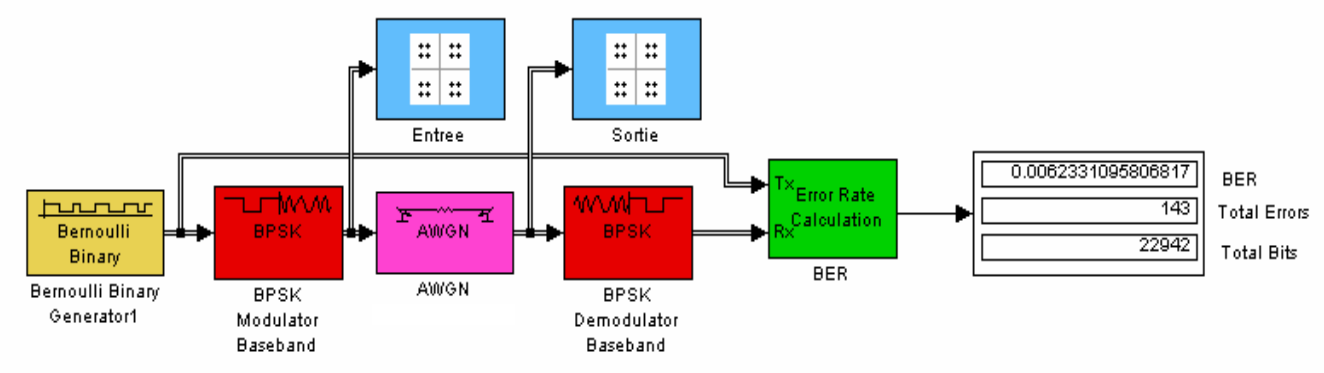

Figure 4 - Modélisation de la chaîne de modulation BPSK sur canal AWGN.

\subsection{Modélisation de la chaîne de modulation démodulation et de mise en forme}

Une fois la chaîne de modulation et démodulation modélisée, nous proposons aux étudiants de positionner un filtre de mise en forme (adapté). Tout d'abord ils utilisent un filtre de type porte, puis un filtre de type racine de cosinus surélevé (l'intérêt du type de filtre à utiliser est enseigné dans les cours de communications numériques du semestre 4). Ainsi les étudiants voient le principe et l'action de chacune de ces mises en forme dans la lutte contre l'interférence entre symboles (IES).

En effet la présence d'IES, qui contient l'information utile sur plusieurs symboles émis, ne permet pas s d'effectuer une décision symboles par symboles qui soit optimale. Pour résoudre il faut faire en sorte que ce terme soit nul, on parle alors de canal de Nyquist.

Critère de Nyquist : Supposons que les symboles soient statistiquement indépendants et que le filtre adapté soit suffisamment fin, alors le terme d'IES est nul et l'échantillon prélevé à l'instant $(n T+\tau)$ ne dépend que du symbole émis à l'instant $n T$. Cette condition s'appelle condition de Nyquist et elle conduit à un canal idéal de Nyquist. Pour que cette condition soit vérifiée, il faut que le débit symbole $R$, sur un canal de bande $B$ vérifie : $R \leq 2 B$. Le cas limite correspond à un filtre rectangulaire qui n'est pas réalisable. Aussi une forme largement utilisée est celle des impulsions dites enede cosinus surélevé. L'utilisation d'un filtre en racine de cosinus surélevé (dit 1/2 Nyquist) à l'émission et à la réception permet de réaliser sur l'ensemble de la chaîne DVB-S permet de réaliser une forme d'impulsion de Nyquist.

Ils prennent de plus conscience que les filtres de mise en forme introduisent un retard de propagation dans la chaîne, égal à la somme de la moitié de la longueur des deux filtres (causalité des filtres). Il leur est donc nécessaire de prendre en compte ce retard pour obtenir une mesure exacte du TEB en simulation. Si on considère les deux filtres de longueur $N$, alors le retard introduit sera de $N=\frac{N}{2}+\frac{N}{2}$.

La figure 5 donne un exemple de modélisation avec une modulation de type QPSK et un filtrage de mise en forme de type racine de cosinus surélevé. Sur ce modèle le bloc de calcul du taux d'erreur tient compte du retard apporté par le filtre de mise en forme. 


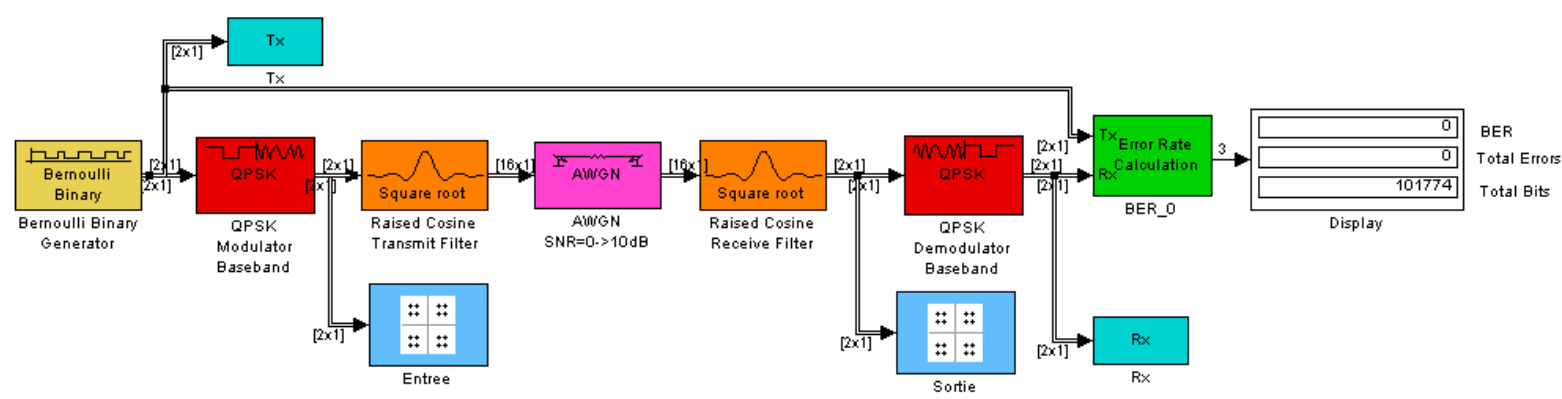

Figure 5 - Modélisation de la chaîne de modulation QPSK avec la mise en forme (filtre en racine de cosinus surélevé).

\subsection{Introduction d'un code correcteur d'erreurs dans la chaîne de transmission : codeur convolutif et décodeur de Viterbi}

Il est important de signaler aux étudiants l'intérêt d'un tel codeur/décodeur, qui permet de corriger en réception d'éventuelles erreurs de transmission. Le but ici n'étant pas de montrer en détail l'algorithme de Viterbi mais de faire comprendre aux étudiants à l'aide d'un exemple simple comment le calcul des métriques et la sélection du chemin le plus probable à la fin de l'algorithme de Viterbi permet de corriger certaines erreurs de transmission.

A partir de cette étape les étudiants n'utilisent que la modulation du type QPSK pour se focaliser sur la norme DVB-S. L'introduction d'un décodeur de Viterbi est délicate puisqu'elle engendre un retard supplémentaire qu'il faut déterminer pour la configuration du bloc de détermination du TEB. La fenêtre glissante utilisée pour le décodage de Viterbi ayant une profondeur de $n T_{b}$, avec $T_{b}$ le temps bit, on doit avoir en théorie un retard de $n$ bits en sortie du décodeur (la profondeur de la fenêtre glissante se règle dans les options du bloc Viterbi Decoder). Ici le retard introduit par les filtres de mise en forme s'effectue sur les bits codés. Or le rendement du codeur étant de $1 / 2$ (sans poinçonnage), le retard sur les bits non codés du au filtrage est donc de $N / 2$ bits. Ainsi pour la synchronisation du paquet émis Tx avec le paquet Rx, il faudra cette fois-ci paramétrer un retard total de $(N / 2+n)$ bits dans le bloc Error Rate Calculation. Pour vérifier le retard introduit par le décodeur de Viterbi, on place un buffer en entrée $T x$ et en sortie $R x$ de la chaine en fixant un SNR assez élevé pour assurer une transmission sans erreur. La figure 6 donne la modélisation de la chaîne avec le codeur convolutif au niveau de l'émission et le décodeur de Viterbi au niveau de la réception.

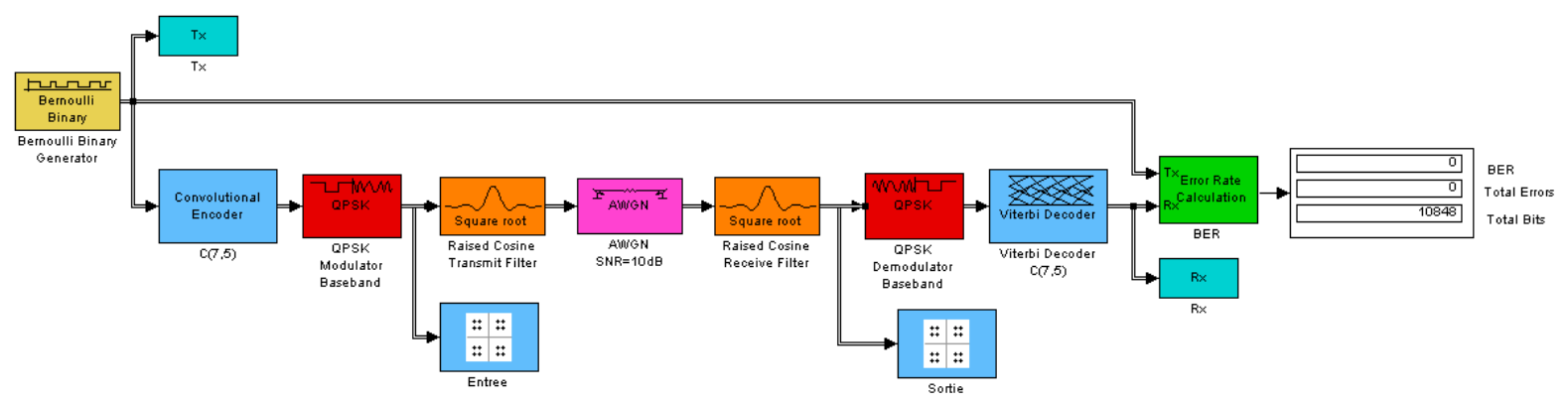

Figure 6 - Modélisation de la chaîne avec le codeur convolutif et le décodeur de Viterbi.

\subsection{Etude du codage de Reed-Solomon pour la détection et la correction des erreurs de transmission}

Le point le plus délicat lorsque l'on fait de la modélisation sous Simulink est de synchroniser correctement les paquets de données pour que les décodeurs qui fonctionnent par paquet de données ou les blocs qui évaluent les taux d'erreurs ne donnent pas de résultats erronés. 
Dans la norme DVB-S c'est le codeur de Reed-Solomon (RS) qui est utilisé comme code détecteur et correcteur d'erreurs. Conformément à la norme, ce codeur traite des paquets de longueur 188 octets et y ajoute 16 octets de redondance, soit 204 octets en sortie du codeur. En réception si les données sont retardées (par les autres éléments de la chaîne, comme les filtres de mise en forme et le décodeur de Viterbi) alors il est nécessaire de synchroniser le début du paquet émis avec le début du décodage de ce dernier, auquel cas le décodage serait catastrophique car les octets de redondance se trouveraient considérés comme des octets d'informations et les premiers octets du paquet suivant comme octets de redondance du premier. Ce phénomène est illustré au travers de la figure 7 suivante :

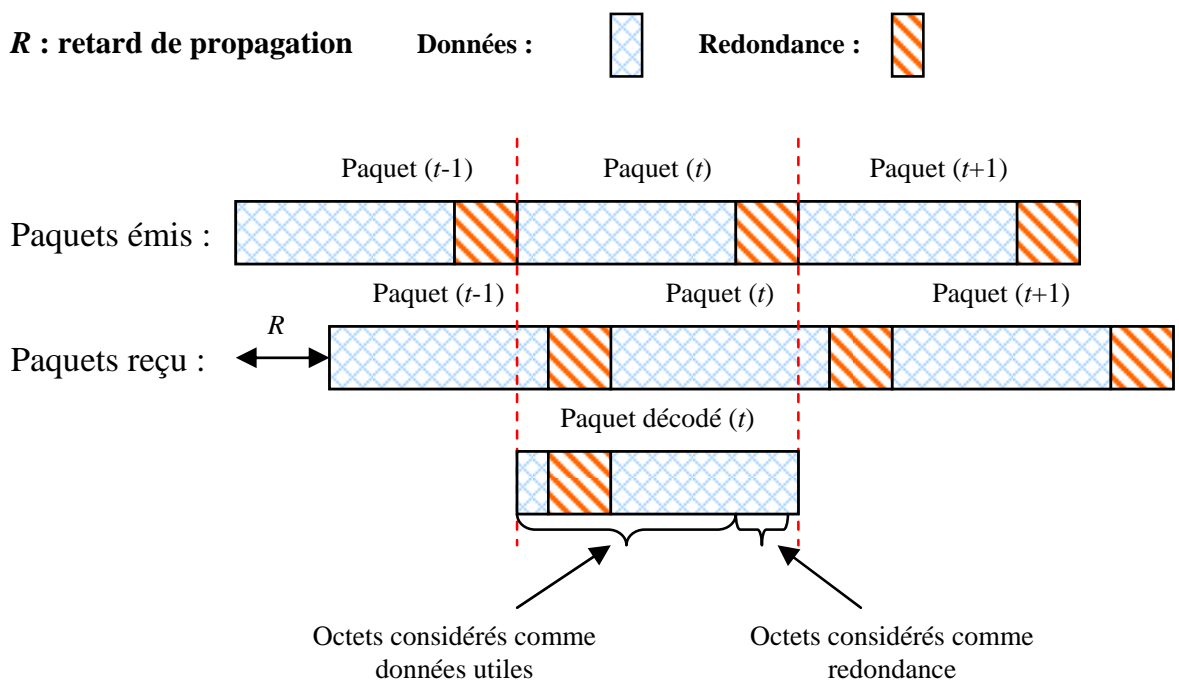

Figure 7 - Importance de la synchronisation d'une transmission par paquet.

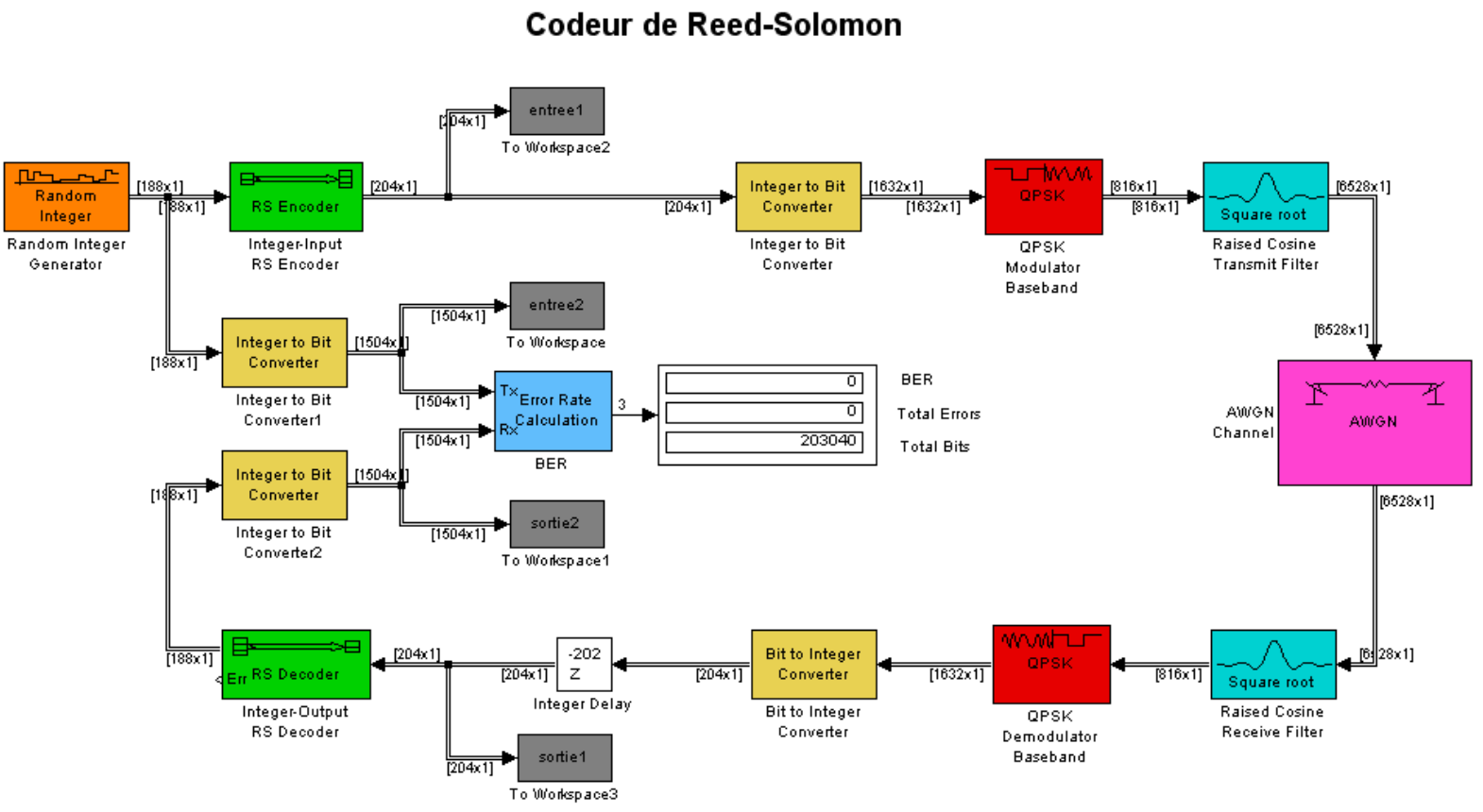

Figure 8 - Modélisation de la chaîne avec le codeur et décodeur RS.

Il est donc nécessaire de synchroniser les données avant l'entrée du décodeur. C’est ce que l'on peut voir sur la figure 8 qui illustre la modélisation d'une chaîne de transmission utilisant un codeur/décodeur RS, un filtrage de mise en forme en racine de cosinus surélevé et une modulation QPSK. En réception, après la démodulation, on apporte un retard de 202 octets supplémentaires afin de synchroniser correctement le début du premier paquet. En effet, dans les conditions de simulations de la figure 7, le retard total introduit par le filtrage est de 2 octets. Ainsi, l'ensemble de la 
transmission se retrouve retardé en réception de 204 octets (soit un paquet) permettant de synchroniser le décodeur RS.

\subsection{Principe de l'entrelaceur/désentrelaceur convolutif}

A l'émission, les octets d'un paquet sont répartis dans d'autres paquets, ce qui permet d'éviter d'avoir à corriger une longue suite de bits (ou octets) faux consécutifs. L'entrelacement permet de répartir les erreurs sur plusieurs paquets, facilitant ainsi la détection et correction d'erreurs du décodeur RS.

Le principe de l'entrelaceur convolutif utilise plusieurs registres à décalages qui vont induire un retard. L'entrelaceur utilise deux paramètres : le nombre de branches $K$ et la profondeur $T$ (en octet) du registre à décalage de base.

Nous illustrons le principe de l'entrelaceur dans un cas simple, sur la figure 9, 3 branches et un registre à décalage de base, de profondeur 2 octets.

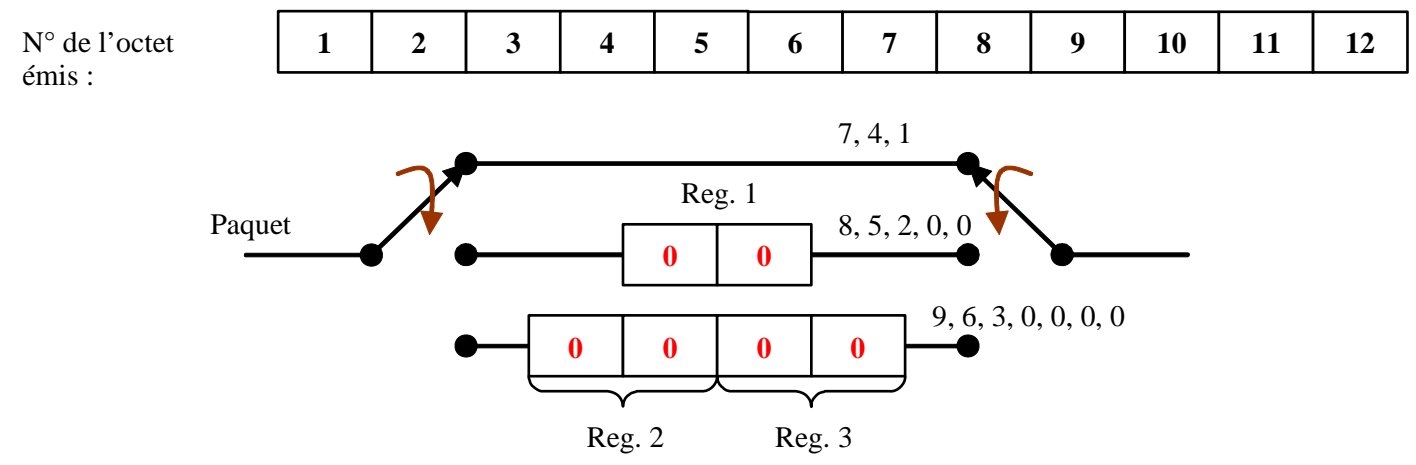

\begin{tabular}{l|l|l|l|l|l|l|l|l|l|l|l|l|l|} 
Après entrelacement : & $\mathbf{1}$ & $\mathbf{0}$ & $\mathbf{0}$ & $\mathbf{4}$ & $\mathbf{0}$ & $\mathbf{0}$ & $\mathbf{7}$ & $\mathbf{2}$ & $\mathbf{0}$ & $\mathbf{1 0}$ & $\mathbf{5}$ & $\ldots$ \\
\hline
\end{tabular}
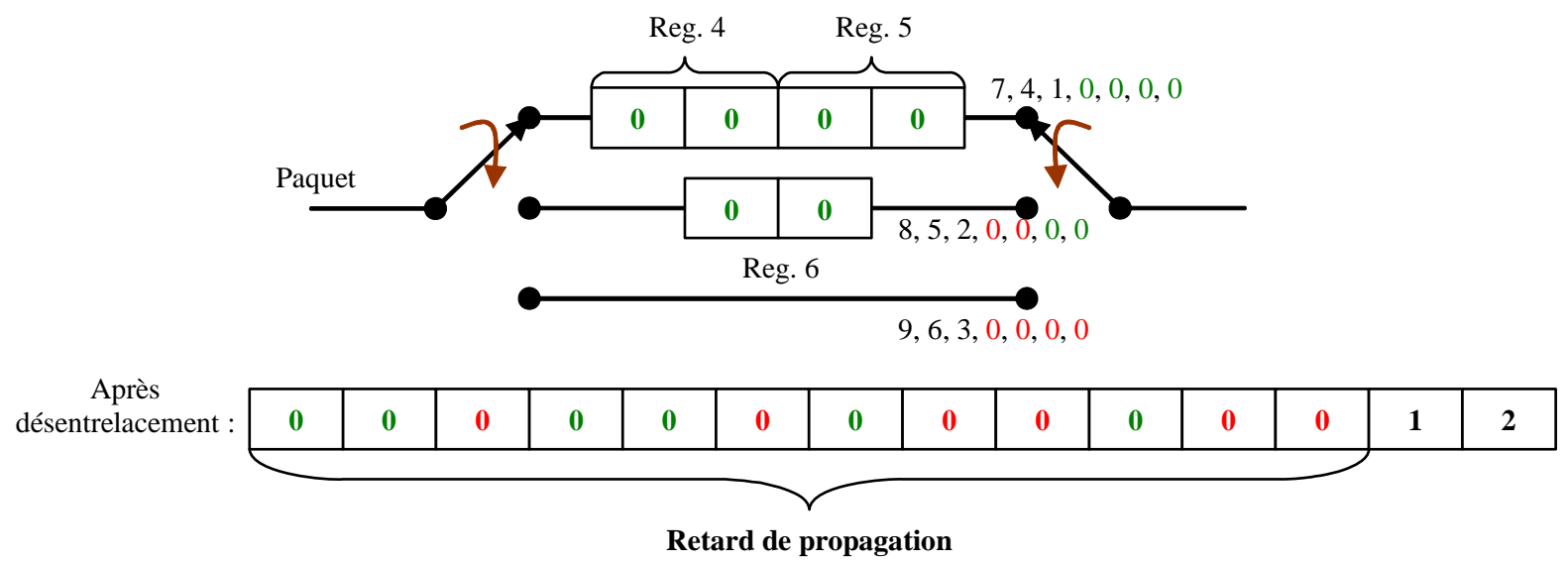

Figure 9 - Processus d'entrelacement/désentrelacement à l'initialisation, avec $K=3$ et $T=2$.

Celui utilisé dans la norme DVB-S est constitué de 12 branches et chacune d'entre elle est composée de registres à décalage multiples de 17 octets. Les figures 10-a et 10-b schématisent respectivement le fonctionnement de l'entrelaceur convolutif $(12,17)$ et du désentrelaceur $(12,17)$.

En effet, chaque branche est constituée d'un multiple de 17 octets : de 17 octets pour la première branche à $17 \times 11$ octets pour la dernière branche. Ainsi, en sortie du désentrelaceur convolutif $(12,17)$ le début du premier paquet entrelacé arrive avec un retard de $12 *(17 * 11)=2244$ octets, ce qui équivaut au nombre de registres multiplié par la taille maximale du plus grand registre (temps nécessaire pour que les registres soient totalement remplis à l'initialisation de la chaîne). Ce délai sera pris en compte dans notre simulation pour le calcul du taux d'erreur binaire. 
Plus généralement, pour un entrelaceur/désentrelaceur $(K, T)$, le retard en octet à considérer pour évaluer le taux d'erreur binaire est de $K \times(K-1) \times T$.

Il faut donc comme pour le cas de codage de RS, synchroniser correctement le début du paquet à désentrelacer en réception pour ne pas avoir de paquet complètement faux en entrée du décodeur de RS. C'est pour cela qu'on est obligé d'insérer une ligne de retard juste avant le bloc Convolutional Deinterleaver sur la figure 11.

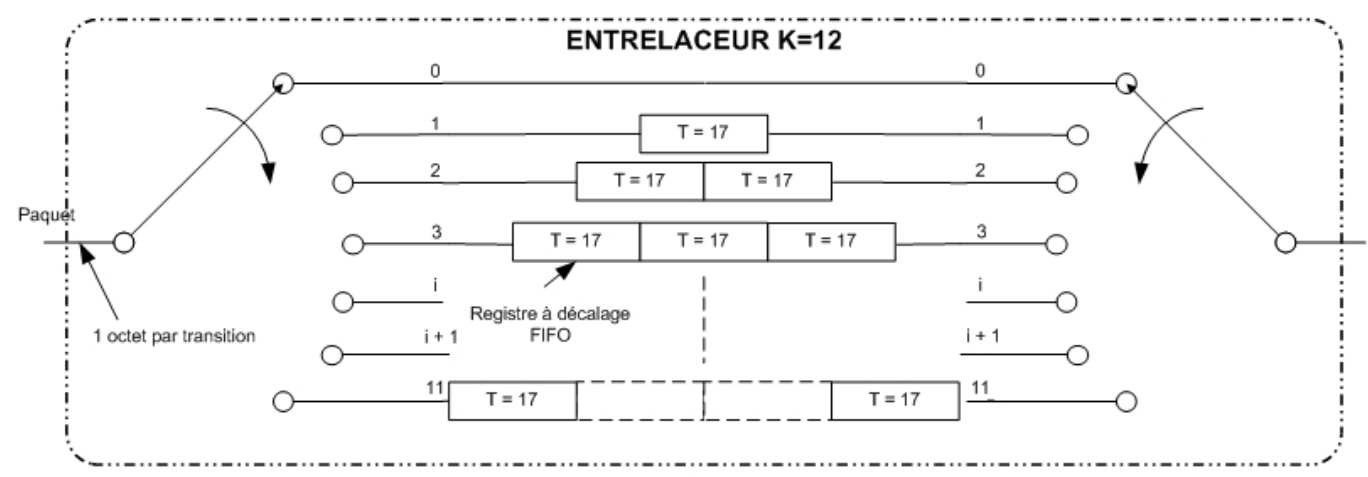

10 -a) entrelaceur $(12,17)$

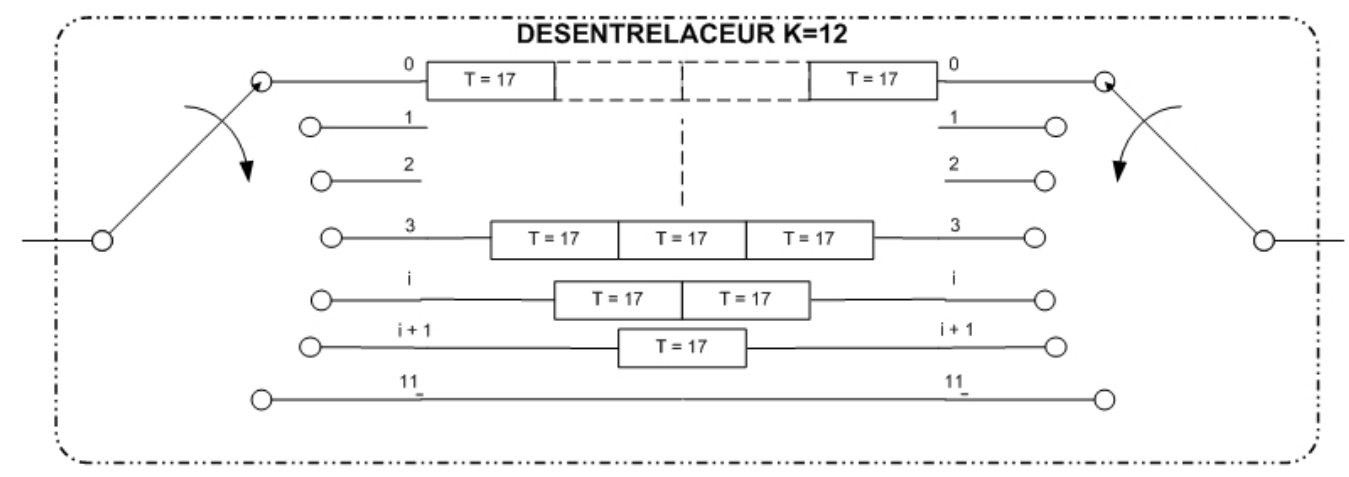

10-b) désentrelaceur $(12,17)$

Figure 10 - Entrelaceur/Désentrelaceur (12, 17) constituant une chaîne DVB-S.

\subsection{Modélisation de chaîne complète DVB-S}

Dans cette dernière partie, tous les blocs de la chaine d'émission-réception sont modélisés, y compris l'entrelaceur que nous n'avions pas pour l'instant dans les modélisations successives. La figure 11 donne le modèle complet de la chaîne d'émission-réception DVB-S.

Notez que pour améliorer le rendement du codeur convolutif, un poinçonneur, poinçonnant de façon déterminée les voix I et Q [4], peut être placé en sortie de ce dernier. Dans notre cas (cf. figure 11), nous nous sommes contentés d'un rendement de $1 \frac{1}{2}$. 


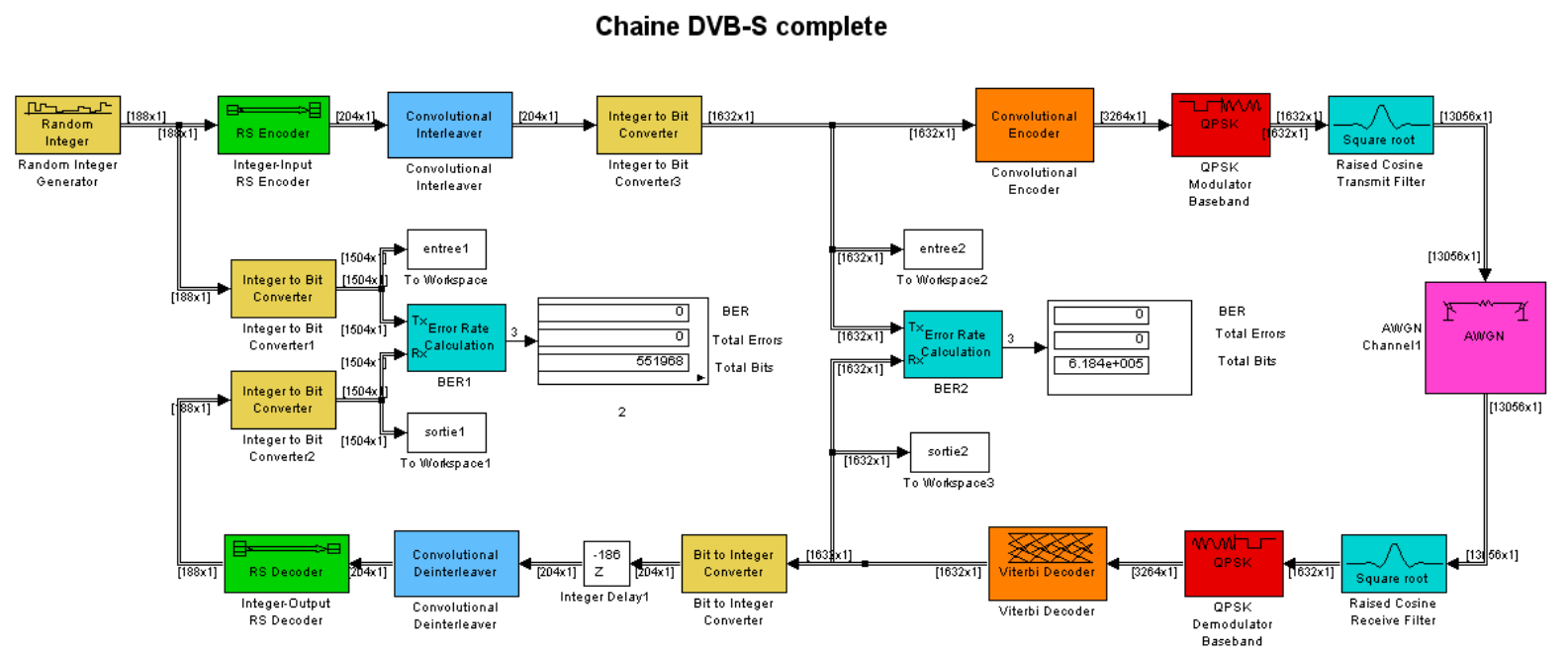

Figure 11 - Modélisation de la chaîne DVB-S complète.

Une fois la chaîne complètement synchronisée, les performances de la modélisation sont effectuées et les résultats de simulation sont présentés sur la figure 12. Ceux-ci sont bien conformes à la théorie.

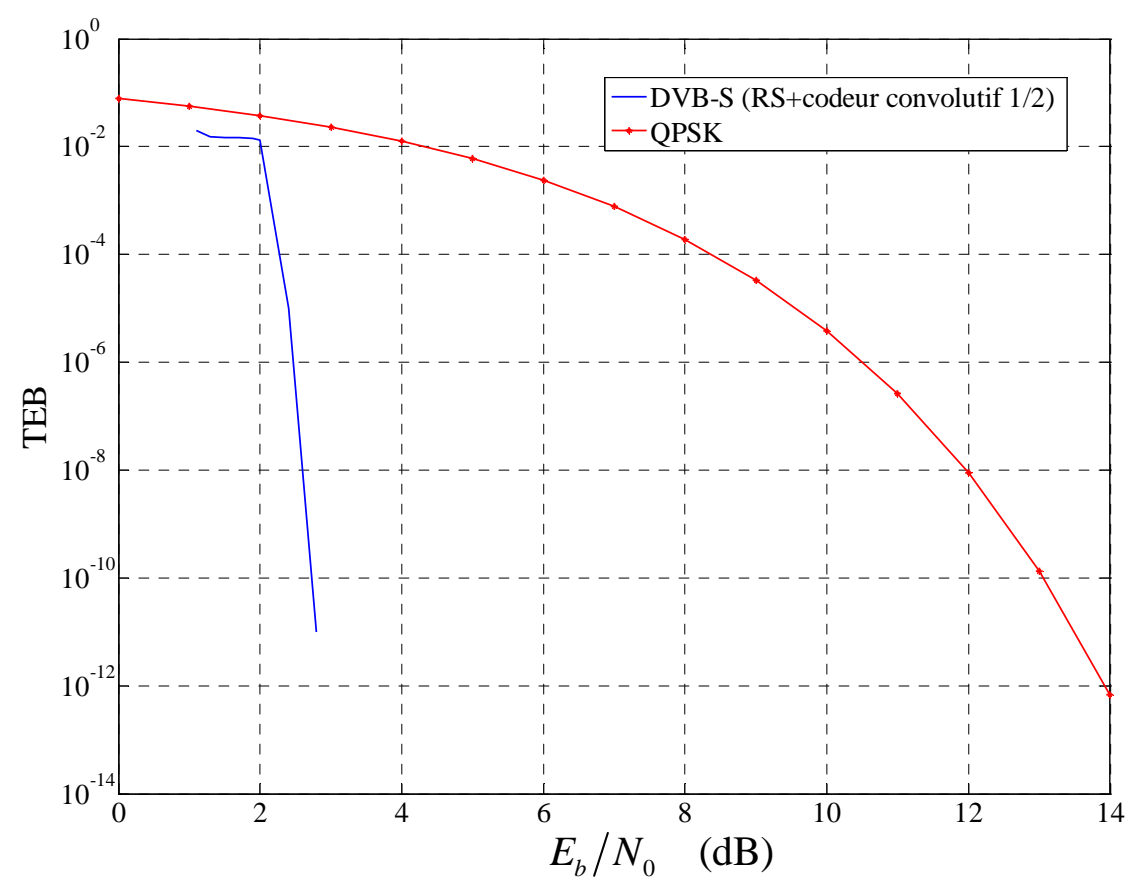

Figure 12 - Taux d'erreur binaire d'une chaîne DVB-S de rendement 1⁄2

\section{Conclusion}

Depuis deux années, nous proposons aux étudiants de l'option SRT du département électronique de l'ENSEIRB, un projet de modélisation de la chaîne DVB-S avec l'outil logiciel SIMULINK. Ce projet permet aux étudiants d'acquérir une connaissance précise de la norme DVB-S. Il leur permet également d'utiliser leurs connaissances en communications numériques et radiocommunications pour mettre en œuvre de façon efficace et cohérente les différents blocs constituants la chaîne comme le Reed-Solomon, le codeur convolutif ou encore l'entrelaceur/désentrelaceur. De plus, ce projet prépare 
ces futurs ingénieurs à la compréhension d’une norme, compétence opérationnelle essentielle de l’ingénieur.

De part la nature du sujet et l'approche que nous proposons, nous observons un vif intérêt des étudiants pour ce projet. Ils prennent conscience de la difficulté de la tâche qui les attend lorsqu'ils seront en situation professionnelle.

Devant la réussite de ce projet la première année, nous l’avons donc reconduit mais aussi proposé à d'autres options de spécialité de l'ENSEIRB. Cette année ce projet est aussi proposé aux étudiants en spécialité ISNC (Ingénierie des Systèmes Numériques de Communication) du département télécommunications.

\section{Remerciements}

Nous tenons à remercier les étudiants des promotions 2007 et 2008 de la spécialité SRT du département électronique de l'ENSEIRB, qui par leurs questions et remarques nous ont permis d'améliorer notre proposition pédagogique. Nous remercions plus particulièrement Melle Nathalie Flambard et M. Sébastien Villette dont le rapport a été utilisé pour rédiger cet article.

\section{Bibliographie}

\section{[1] http://www.etsi.org/WebSite/Technologies/DVBS.aspx}

\section{[2] http://www.dvb.org/}

\section{[3] http://www.enseirb.fr}

[4]European Telecommunication Standards Institute. "Digital Vidéo Boadcasting (DVB) ; Framing structure, channel coding modulation for 11/12 GHz satellite service". European Standard (Telecommunications series), EN 300421 v1.1.2, August 1997.

[5] Sergio Isla Hernandez. "Simulation and Evaluation of a DVB System using Simulink". Bachelor thesis, Departement of Electronics Systems at Linköping Institue of Technology, Suede, April 2005.

[6]European Telecommunication Standards Institute. "Digital Vidéo Boadcasting (DVB) ; Framing structure, channel coding modulation for cable system". European Standard (Telecommunications series), EN 300429 v1.1.2, April 1998.

[7]European Telecommunication Standards Institute. "Digital Vidéo Boadcasting (DVB) ; Framing structure, channel coding modulation for digital terrestrial television". European Standard (Telecommunications series), EN 300744 v1.1.2, August 1997.

[8] http://www.mathworks.com/access/helpdesk/help/toolbox/comm/comm.shtml

Lilian Bossuet a obtenu le diplôme d'ingénieur en électronique de l'ENSEA en 1999, l'agrégation génie électrique en 2000 à l'ENS Cachan, le DEA d'électronique de l'INSA de Rennes en 2001 et le Doctorat en Sciences et Sciences de l'Ingénieur de l'Université de Bretagne Sud à Lorient en 2004. Il a effectué sa thèse au laboratoire LESTER à Lorient sur le thème de l'exploration de l'espace de conception des architectures reconfigurables. Après une année d'ATER à l'Université de Bretagne Sud et un séjour de recherche à l'Université du Massachussetts aux USA, il a rejoint en 2005 en tant que Maître de Conférences le laboratoire IMS et l'ENSEIRB à Bordeaux ou il enseigne principalement l'électronique numérique. Ses recherches au département COFI de l'IMS portent sur la sécurité matérielle et logicielle des systèmes embarqués, les architectures et systèmes reconfigurables, l'implémentation d'applications du traitement du signal dans des FPGA et la compensation des non-linéarités des CAN.

Guillaume Ferré a obtenu le diplôme en électronique et télécommunications de l'ENSIL en 2003, le DEA de télécommunications, hautes fréquences et optoélectronique de l'Université de Limoges en 2003 et le Doctorat en électronique des hautes fréquences et optoélectronique de l'Université de 
Limoges en 2006. Il a effectué sa thèse au laboratoire Xlim de Limoges sur le codage de canal et les techniques de décodage itératives pour les systèmes sans fil multi-antennes. Après une année en tant qu'ATER à l'ENSIL, une année de post-doctorat au laboratoire IMS de Bordeaux, il rejoint en 2008 l'ENSEIRB en tant que Maître de Conférences. Ses activités de recherches en signal, au département LAPS de l'IMS, sont axées sur le codage de canal, les techniques d'accès des systèmes multi-antennes et la géolocalisation. 